\title{
BMJ Open Efficacy of mobilisation with movement in chronic shoulder pain: protocol for a systematic review and meta-analysis of controlled trials
}

\author{
Arnstein Storås, ${ }^{1}$ Fabian Lillebostad, ${ }^{1}$ Sturla Haslerud, ${ }^{2}$ Jon Joensen, ${ }^{1}$ \\ Jan Magnus Bjordal, ${ }^{1}$ Martin Bjørn Stausholm (i) ${ }^{1}$
}

To cite: Storås A, Lillebostad $F$, Haslerud S, et al. Efficacy of mobilisation with movement in chronic shoulder pain: protocol for a systematic controlled trials. BMJ Open 2021;11:e049563. doi:10.1136/ bmjopen-2021-049563

- Prepublication history and additional supplemental material for this paper are available online. To view these files, please visit the journal online (http://dx.doi.org/10.1136/ bmjopen-2021-049563)

Received 28 January 2021 Accepted 09 August 2021 review and meta-analysis of

\section{ABSTRACT}

Introduction Shoulder pain affects approximately one in four adults and is thus one of the most common musculoskeletal problems. Only $50 \%$ of patients who begin treatment for shoulder pain are cured within 6 months. There is a need for systematic reviews to estimate the effectiveness of shoulder treatments. We decided to evaluate the effect of mobilisation with movement (MWM) on chronic shoulder pain in a systematic review.

Methods and analysis The review will include controlled trial articles identified via five electronic databases (PubMed, Embase, Cumulative Index to Nursing and Allied Health Literature, Physiotherapy Evidence Database and Cochrane Central Register of Controlled Trials), reference lists, citations searches and experts in the field. Only controlled trials involving participants with a mean duration of pain of $\geq 3$ months, in which the effectiveness of MWM has been compared with non-invasive treatments, sham mobilisation or wait-and-see will be included. The included trials will be synthesised with random effects meta-analyses. Risk-of-bias will be assessed with the Physiotherapy Evidence Database 0-10 point scale.

Ethics and dissemination The review does not require ethics approval as it is based on anonymised data from trial reports. The results of the review will be disseminated through a peer-reviewed publication.

PROSPERO registration number CRD42018109380.

\section{INTRODUCTION}

(C) Author(s) (or their employer(s)) 2021. Re-use permitted under CC BY-NC. No commercial re-use. See rights and permissions. Published by BMJ.

${ }^{1}$ Department of Global Public Health and Primary Care, University of Bergen, Bergen, Norway

${ }^{2}$ Department of Public Health, University of Stavanger, Stavanger, Norway

Correspondence to Martin Bjørn Stausholm; m.b.stausholm@gmail.com
Shoulder pain currently affects $18 \%-26 \%$ of adults, making it one of the most common musculoskeletal problems. ${ }^{1}$ The symptoms involved are often persistent and disabling and the reason for sick leave. ${ }^{1}$ Although there are numerous ways shoulder disorders occur, atraumatic shoulder disorders, including subacromial pain syndrome (SAPS) and adhesive capsulitis (AC), predominate in numbers. ${ }^{1}$

SAPS involves pain in the acromial area and it is typically worsening while lifting the arm. The pathology is sometimes referred to as shoulder impingement syndrome, however,
Strengths and limitations of this study

- This review will be conducted in conformance with a detailed a priori published protocol, which includes, for example, a plan for estimating standard deviations for the meta-analyses.

- We will search for eligible trial articles using five electronic literature databases, reference lists of relevant trial and review reports, citations and experts in the field.

- The selection of trials, risk-of-bias assessment and data extraction will be handled in a two-person procedure.

Only studies reported in English and Nordic languages will be considered for inclusion.

- There will be no comparisons between shoulder mobilisation with movement and surgery.

recent studies show that it is not associated with impingement of tissues, and hence SAPS is a better term for it. ${ }^{2}$ SAPS is the most common shoulder disorder, accounting for approximately $36 \%$ of all shoulder diagnoses. ${ }^{3}$ The risk of SAPS is increased by smoking, sleeping in the decubitus position ${ }^{4}$ and overhead sports, such as handball play. ${ }^{5}$

$\mathrm{AC}$ is an idiopathic inflammatory shoulder disorder with excessive adhesive (scar) formation and restricted range of motion. The prevalence of AC has been estimated to be $2 \%-5 \%$ in the general population and $13.4 \%$ in persons with diabetes mellitus. ${ }^{6}$ Furthermore, AC most frequently occurs in persons aged 40-60 years and especially in females. ${ }^{7}$

Unfortunately, only $50 \%$ of patients who begin a course of treatment for shoulder pain are cured within 6 months. ${ }^{8}$ There is currently a debate going on about whether shoulder examination should focus on finding a specific anatomical structure as a pain generator. Other authors have developed non-specific (non-anatomical) examination 
systems with treatment algorithms. One of these systems is using broader terms to classify patients in one out of three categories: irritable SAPS, non-irritable SAPS and degenerative SAPS. ${ }^{9}$ In this system, only the irritable SAPS group is offered local treatment like cryotherapy to reduce pain and irritation.

Manual therapy is also suggested as a pain-relieving treatment option in this system. Manual therapy is used in the treatment of peripheral joint pain, and Brian Mulligan's technique mobilisation with movement (MWM) is one variant. According to Mulligan, the correction of positional faults in and around the joint by MWM may lead to a resolution of the symptoms..$^{10}$ The technique for peripheral joints combines continuous manual application of 'gliding' force to a joint, with the purpose of correcting these positional faults with simultaneous physiological movement of the joint, performed actively by the patient or passively by the therapist. To achieve a desired result, many repetitions of painless MWM are needed, sometimes combined with overpressure from the therapist. It has been argued that correct application of these techniques would cause the patient's pain on movement to disappear immediately after the first treatment session. ${ }^{10}$ Mulligan's original 'positional fault' theory for the effect of MWM was based on a notion of 'misalignment' between the joint's articular surfaces, which arise secondary to an injury and cause an incorrect glide in the joint. This would produce pain, stiffness and/or weakness. However, this biomechanical theory has not been verified..$^{10}$ It has been hypothesised that the effects of MWM are neurophysiological. Mechanoreceptors are excitated in the joint capsule, which in turn modulates central nervous actions by inhibiting incoming nociceptive information. This 'gate control' mechanism suppresses pain. In addition, stimulation of other centres, such as the dorsal periaqueductal grey matter region, produces a deep and selective analgesia. ${ }^{11} \mathrm{~A}$ more recently proposed theory is that pain reduction by MWM concerns habituating and excitation. According to this theory, patients who experience pain during movement may develop fear-avoidance behaviour. Consequently, progressive exposure to the feared movements may desensitise the nervous system through actions of habitation. $^{12}$

The effectiveness of MWM on peripheral joint pain has been systematically reviewed by Hing et al, Westad et al and Stathopoulos et al. ${ }^{13-15}$ Several relevant trials have been published after the review by Hing et al and yet the review by Westad et al only includes a single trial on shoulder pain. ${ }^{13}$ Only one investigator selected the trials for the review by Westad $e a^{13}$ and this may be the reason why it lack some eligible trials. Only the review by Stathopoulos et al features a meta-analysis, however, they systematically excluded all relevant trials published before year 2008. ${ }^{15}$ Therefore, we decided to conduct a new systematic review and meta-analysis on the topic, and we decided to focus on chronic pain of the shoulder due to the complexity of this joint. We will use the latest definition of chronic pain adopted by WHO, that is, pain for at least 3 months. ${ }^{16}$ Our

\begin{tabular}{|c|c|}
\hline $\begin{array}{l}\text { PICOS } \\
\text { strategy }\end{array}$ & Inclusion criteria \\
\hline Participants & $\begin{array}{l}\text { Persons with shoulder pain in the } \\
\text { previous } \geq 3 \text { months }\end{array}$ \\
\hline Intervention & Mobilisation with movement \\
\hline Comparison & $\begin{array}{l}\text { Conservative treatment, sham mobilisation } \\
\text { or no treatment }\end{array}$ \\
\hline Outcomes & $\begin{array}{l}\text { Pain, self-reported disability, quality of life } \\
\text { and/or shoulder range of motion }\end{array}$ \\
\hline Study design & Controlled trials \\
\hline
\end{tabular}

PICOS, Participants, Intervention, Comparison, Outcomes, Study Design.

hypothesis is that MWM can reduce pain and disability and increase quality of life (QoL) and shoulder range of motion in persons with chronic shoulder pain.

\section{METHODS}

This protocol is reported in accordance with the Preferred Reporting Items of Systematic Reviews and Meta-Analyses for Protocols 2015. ${ }^{17}$ The search for eligible trial articles is ongoing and the review is expected to be completed by 1 October 2021.

\section{Literature search and selection of studies}

We will include controlled trials involving participants with mean duration of shoulder pain of $\geq 3$ months, in which the effectiveness of MWM was compared with that of other conservative treatment, sham mobilisation or wait-and-see in terms of pain, self-reported disability, QoL and/or shoulder range of motion. A summary of the eligibility criteria is provided in table 1 .

A search for eligible articles indexed in PubMed, Embase and Physiotherapy Evidence Database, Cumulative Index to Nursing and Allied Health Literature and Cochrane Central Register of Controlled Trials was performed on 25 March 2020. The database search strings included synonyms for MWM and shoulder pain. Keywords were added to the search string when optional. The PubMed search string is provided in online supplemental material. The search will be continued by reading reference lists of the eligible trial and relevant review articles, citations and involvement of experts in the field. Only studies reported in English and Nordic languages will be considered for inclusion.

Two reviewers will each independently select the trial articles. Both reviewers will read the titles/abstracts of all the records identified in the search, and any obtainable full-text article will be retrieved if it is judged potential eligible by a reviewer. Both reviewers will evaluate the full texts of all the potentially eligible retrieved articles and make an independent decision to include or exclude each article, with close attention to the eligibility criteria. Selection disagreements will be resolved by discussion. 
Any retrieved ineligible article will be excluded with an explicit reason why.

\section{Risk-of-bias analysis}

The included trials will be assessed for risk-of-bias at the outcome level by two independent reviewers using the Physiotherapy Evidence Database $0-10$ point scale ${ }^{18}$ as it has been found to be a valid and reliable tool. ${ }^{19}$ Riskof-bias disagreements will be resolved by discussion. The reasons for our risk-of-bias scorings of the included trials will be stated.

Likelihood of publication bias will be assessed with graphical funnel plots if at least 10 trials are available. ${ }^{20}$

\section{Data extraction and meta-analysis}

Extraction of the following information will be mandatory: year of publication, number of participants randomised to MWM and control groups, type and duration of interventions, time points of assessments, participant characteristics (age, gender, body mass index, baseline shoulder pain), effect estimates (pain, disability, QoL and shoulder range of motion) and adverse events.

The data extraction will be handled by two reviewers. One reviewer will extract the data independently and another reviewer will check the work for correctness. Data disagreements will be resolved by discussion.

The primary outcome is pain intensity and the secondary outcomes are self-reported disability and QoL and shoulder range of motion. Outcomes of the same nature reported on different scales will be meta-analysed using the Hedges' $g$ standardised mean difference (SMD). The SMD will be interpreted as follows: SMDs of $0.2, \sim 0.5$ and $>0.8$ represent a small, moderate and large difference, respectively. ${ }^{20}$

Outcomes of the same nature reported on the same scale will be meta-analysed using the mean difference method.

The DerSimonian and Laird version of the random effects meta-analysis model will be utilised. Impact from heterogeneity (inconsistency) on the analyses will be examined using $\mathrm{I}^{2}$ statistics. $\mathrm{I}^{2}$ values range from $0 \%$ to $100 \%{ }^{20}$ The $\mathrm{I}^{2}$ values will be categorised as low $(25 \%)$, moderate $(50 \%)$ and high $(75 \%) .{ }^{21}$

The standard deviations for meta-analysis will be extracted or estimated from other variance data in the following order: (1) standard deviation, (2) standard error, (3) 95\% confidence interval, (4) p value, (5) interquartile range, (6) median of correlations, (7) visually from graph or (8) other methods. ${ }^{20}$

The trials may be subgrouped by shoulder pathology, risk of bias, MWM protocols and comparisons.

\section{Patient and public involvement}

There was no patient or public involvement in the development of this protocol.

\section{Amendments}

Any amendment to this protocol will be reported in the result article.

\section{Dissemination}

The results of the review will be disseminated through peer-reviewed publication.

Contributors AS, JJ and MBS registered the methods on the PROSPERO website and drafted the manuscript. The manuscript was subsequently revised for critical intellectual content by AS, FL, SH, JJ, JMB and MBS.

Funding The University of Bergen funded this research.

Disclaimer No specific grant from any funding agency in the public, commercial or not-for-profit sectors was received for this work.

Competing interests None declared.

Patient consent for publication Not required.

Provenance and peer review Not commissioned; externally peer reviewed.

Supplemental material This content has been supplied by the author(s). It has not been vetted by BMJ Publishing Group Limited (BMJ) and may not have been peer-reviewed. Any opinions or recommendations discussed are solely those of the author(s) and are not endorsed by BMJ. BMJ disclaims all liability and responsibility arising from any reliance placed on the content. Where the content includes any translated material, BMJ does not warrant the accuracy and reliability of the translations (including but not limited to local regulations, clinical guidelines, terminology, drug names and drug dosages), and is not responsible for any error and/or omissions arising from translation and adaptation or otherwise.

Open access This is an open access article distributed in accordance with the Creative Commons Attribution Non Commercial (CC BY-NC 4.0) license, which permits others to distribute, remix, adapt, build upon this work non-commercially, and license their derivative works on different terms, provided the original work is properly cited, appropriate credit is given, any changes made indicated, and the use is non-commercial. See: http://creativecommons.org/licenses/by-nc/4.0/.

\section{ORCID iD}

Martin Bjørn Stausholm http://orcid.org/0000-0001-9869-0705

\section{REFERENCES}

1 Linaker $\mathrm{CH}$, Walker-Bone K. Shoulder disorders and occupation. Best Pract Res Clin Rheumatol 2015;29:405-23.

2 Park SW, Chen YT, Thompson L, et al. No relationship between the acromiohumeral distance and pain in adults with subacromial pain syndrome: a systematic review and meta-analysis. Sci Rep 2020;10:20611.

3 Juel NG, Natvig B. Shoulder diagnoses in secondary care, a one year cohort. BMC Musculoskelet Disord 2014;15:89.

4 Tangtrakulwanich B, Kapkird A. Analyses of possible risk factors for subacromial impingement syndrome. World J Orthop 2012;3:5-9.

5 Oliveira VMAde, Pitangui ACR, Gomes MRA, et al. Shoulder pain in adolescent athletes: prevalence, associated factors and its influence on upper limb function. Braz J Phys Ther 2017;21:107-13.

6 Zreik NH, Malik RA, Charalambous CP. Adhesive capsulitis of the shoulder and diabetes: a meta-analysis of prevalence. Muscles Ligaments Tendons J 2016;6:26-34.

7 Hand C, Clipsham K, Rees JL, et al. Long-term outcome of frozen shoulder. J Shoulder Elbow Surg 2008;17:231-6.

8 Struyf F, Geraets J, Noten S, et al. A multivariable prediction model for the Chronification of non-traumatic shoulder pain: a systematic review. Pain Physician 2016;19:1-10.

9 Lewis J, McCreesh K, Roy J-S, et al. Rotator cuff tendinopathy: Navigating the Diagnosis-Management conundrum. J Orthop Sports Phys Ther 2015;45:923-37.

10 Hing W, Hall T, Mulligan BR. The Mulligan concept of manual therapy: textbook of techniques. 1st edn. Churchill Livingstone, 2014.

11 Baeske R. Mobilisation with movement: a step towards understanding the importance of peripheral mechanoreceptors. Physical Therapy Reviews 2015;20:299-305.

12 Hidalgo B, Pitance L, Hall T, et al. Short-term effects of Mulligan mobilization with movement on pain, disability, and kinematic spinal movements in patients with nonspecific low back pain: a randomized placebo-controlled trial. J Manipulative Physiol Ther 2015;38:365-74.

13 Westad K, Tjoestolvsen F, Hebron C. The effectiveness of Mulligan's mobilisation with movement (MWM) on peripheral joints in musculoskeletal (MSK) conditions: a systematic review. Musculoskelet Sci Pract 2019;39:157-63. 
14 Hing W, Bigelow R, Bremner T. Mulligan's Mobilization with Movement: A Systematic Review. J Man Manip Ther 2009;17:39E-66.

15 Stathopoulos N, Dimitriadis Z, Koumantakis GA. Effectiveness of Mulligan's mobilization with movement techniques on range of motion in peripheral joint pathologies: a systematic review with meta-analysis between 2008 and 2018. J Manipulative Physiol Ther 2019;42:439-49.

16 Treede R-D, Rief W, Barke A, et al. Chronic pain as a symptom or a disease: the IASP classification of chronic pain for the International classification of diseases (ICD-11). Pain 2019;160:19-27.

17 Shamseer L, Moher D, Clarke M, et al. Preferred reporting items for systematic review and meta-analysis protocols (PRISMA-P) 2015: elaboration and explanation. BMJ 2015;349:97647.
18 Physiotherapy evidence database scale, 1999. Available: https:// pedro.org.au/wp-content/uploads/PEDro_scale.pdf [Accessed $26 \mathrm{Jul}$ 2021].

19 Yamato TP, Maher C, Koes B, et al. The PEDro scale had acceptably high convergent validity, construct validity, and interrater reliability in evaluating methodological quality of pharmaceutical trials. $J$ Clin Epidemiol 2017;86:176-81.

20 Higgins JPT, Green S. Cochrane Handbook for systematic reviews of interventions, 2011. Available: http://handbook.cochrane. org/[Accessed 3 Dec 2015].

21 Higgins JPT, Thompson SG, Deeks JJ, et al. Measuring inconsistency in meta-analyses. BMJ 2003;327:557-60. 